\section{Hacer extensión en los pueblos de la Costa de Santa Fe (Argentina): dos experiencias desde la Geografía de los Riesgos}

\author{
María Laura Visintini \\ mlvisintini@yahoo.com.ar \\ (iD) orcid.org/0000-0002-8109-930X \\ Juan Pablo Levrino \\ levrino.juanpablo@gmail.com \\ (iD) orcid.org/0000-0002-5730-0261 \\ Carolina Schmidt \\ carofila@hotmail.com \\ (iD) orcid.org/0000-0002-9716-9595
}

Universidad Nacional del Litoral, Argentina.

\section{Pilar Cabre}

Instituto de Humanidades y Ciencias Sociales del Litoral, CONICET - Universidad Nacional del Litoral, Argentina.

pilargcabre@hotmail.com

(iD) orcid.org/0000-0002-3685-4820
Ambiente y extensión universitaria /

Intervenciones
RECEPCIÓN: 29/06/21

ACEPTACIÓN FINAL: 18/08/21

\section{Resumen}

El presente artículo recupera el trabajo continuo realizado a lo largo de casi diez años en el territorio de los pueblos de la Costa santafesina. En el mismo se reúnen dos instancias de extensión llevadas adelante desde dos marcos institucionales distintos (Proyecto de Extensión de Cátedra y Proyecto de Extensión Universitaria). El área de trabajo comprendió las actuales localidades de Arroyo Leyes y San José del Rincón (departamento La Capital) y Santa Rosa de Calchines, Cayastá, Helvecia, Colonia Mascías y Saladero Cabal (departamento Garay). El objetivo general fue generar y compartir conocimientos que contribuyeran al fortalecimiento de las capacidades territoriales de la población y las instituciones locales frente a las condiciones de riesgo de inundaciones en la zona. En este trabajo, presentamos un abordaje reflexivo sobre las implicancias de dichas prácticas.

Palabras clave: pueblos de la Costa; Santa Fe; extensión universitaria; vulnerabilidad; riesgo ambiental.
Extension in the Towns of the Coast of Santa Fe (Argentina): two experiences from the Geography of Risks

\begin{abstract}
This paper is about the experience of work over almost ten years on the territory of coastal towns of Santa Fe. In this work are collected two instances of extension carried out from different institutional frameworks (a project of extension of cathedra and a project of university extension). The area of work includes the cities of Arroyo Leyes and San Jose of Rincón (department of La Capital) and Santa Rosa de Calchines, Cayastá, Helvecia, Colonia Mascías and Saladero Cabal (department of Garay). The general aim have been to generate and share knowledges that contribute to strengthen the territorial capacities of population and local institution face to risk conditions of the place. In this article we present a reflexive approach about implicancies of those extension practices.
\end{abstract}

Keywords: coastal towns; Santa Fe; university extension; vulnerability; environmental risk.
Extensão nos Municípios do Litoral de Santa Fe (Argentina): duas experiências da Geografia dos Riscos

\section{Resumo}

Este artigo resgata o trabalho contínuo realizado ao longo de quase dez anos no território dos municípios do litoral de Santa Fe. Nele, duas instâncias de extensão articuladas a partir de dois marcos institucionais diferentes (projeto de extensão de cátedras e projeto de extensão universitária). A área de trabalho incluiu os atuais municípios de Arroyo Leyes e San José del Rincón (departamento La Capital) e Santa Rosa de Calchines, Cayastá, Helvecia, Colônia Mascías e Saladero Cabal (departamento Garay). O objetivo geral tem sido gerar e compartilhar conhecimentos que contribuam para o fortalecimento das capacidades territoriais da população e das instituições locais frente a condições de risco de enchentes na zona. Neste trabalho, apresentamos uma abordagem reflexiva sobre as implicações dessas práticas.

Palavras-chave: municípios do litoral; Santa Fe; extensão universitária; vulnerabilidade; risco ambiental.

Para citación de este artículo: Visintini, M. L.; Levrino, J. P.; Schmidt, C. y Cabré, P. (2021). Hacer extensión en los pueblos de la costa de Santa Fe (Argentina): dos experiencias desde la Geografía de los Riesgos. +E: Revista de Extensión Universitaria, 11(15), e0007. doi: 10.14409/ extension.2021.15.Jul-Dic.e0007 


\section{Introducción}

En el presente artículo pretendemos dar cuenta del proceso de trabajo de extensión universitaria llevado a cabo de manera sostenida a lo largo de casi diez años por nuestro equipo de extensionistas. La tarea se centró en el sector sur de los pueblos de la Costa en Santa Fe. El equipo estuvo conformado originalmente por la Dra. Hortensia Castro, Prof. María Laura Visintini, Prof. Carolina Schmidt y los estudiantes Diego Combín, Juan Pablo Levrino, Mónica Resek y Gisela Gatti.

El trabajo se desarrolló a partir de dos proyectos consecutivos correspondientes a diferentes marcos institucionales: por un lado, el Proyecto de Extensión de Cátedra (PEC, 2006-2008), que se inscribía en un programa vigente en la Universidad Nacional del Litoral (UNL) denominado "Universidad y Desarrollo Local y Regional", cuya unidad responsable era la cátedra Introducción a la Geografía del Departamento de Geografía de la Facultad de Humanidades y Ciencias (FHUC-UNL). La institución vinculante elegida para desarrollarlo fue la asociación civil CANOA (Santa Fe).

Por otro lado, la segunda instancia de extensión tenía como marco institucional el Ministerio de Educación de la Nación a través de la convocatoria a proyectos "Un puente entre la universidad y la sociedad". En esta ocasión, participamos de dicha convocatoria con un Proyecto de Extensión Universitaria donde las instituciones participantes eran la UNL (Secretaría de Extensión), la escuela de Educación Media Técnica № 658 María Sánchez de Thompson y la Escuela de Enseñanza Técnica Particular Incorporada № 2020 San Isidro Labrador.

Nuestro interés por dicha área surgió al observar la problemática de las inundaciones, en especial, las que se desarrollaban en el área denominada sector sur de los pueblos de la Costa. Este sector abarca las localidades de San José de Rincón y Arroyo Leyes (en el departamento La Capital) y Santa Rosa de Calchines, Helvecia, Cayastá, Saladero Mariano Cabal y Colonia Mascías (del departamento Garay). La totalidad de los asentamientos y gran parte de las actividades productivas de dicha área se localizan en el albardón, es decir, en un territorio donde el principal peligro natural para la población y sus actividades deviene de los pulsos cíclicos de crecidas del río y de las continuas modificaciones del terreno asociadas a esa dinámica.

Sobre la base de estas experiencias, con este artículo nos proponemos recuperar recorridos, aprendizajes, formas de trabajo y principales actividades llevadas a cabo, desde el presente y de forma reflexiva, a fin de que la sistematización de dichas instancias sea una contribución al campo de la extensión.

Para poder dar cuenta de ello, este trabajo se estructura de la siguiente forma: en primer lugar, se desarrolla el marco teórico utilizado; en segundo lugar, se describen las dos experiencias destacando las acciones de extensión, formación e investigación realizadas; en tercer lugar, se presentan reflexiones en torno a ambas experiencias, donde es central la resignificación del conocimiento académico a partir del trabajo con y para la comunidad que trasciende las formas institucionales.

\section{Marco teórico, enfoques y elección de la problemática}

A partir de la década de 1980 la cuestión ambiental dentro de la Geografía comenzó a ser analizada desde enfoques renovados que incorporaron teorías y métodos provenientes de 
otras ciencias sociales, lo cual produjo una renovación conceptual en el abordaje de la disciplina. La elaboración de nuevas propuestas no deterministas desde la Geografía introdujo la idea de la relación sociedad-naturaleza (en lugar de hombre-medio) a partir de comprender las crisis ambientales como fenómenos sociales, propios de la racionalidad capitalista, y no como limitaciones de la capacidad física del planeta. La Geografía de los Riesgos, como subcampo disciplinar, ofrecía una perspectiva de análisis que permitía pensar en las condiciones históricas que configuraban los desastres, es decir, pensar en el proceso en lugar de poner el foco en el resultado, como lo hacía la perspectiva fisicalista. En tal sentido, empezamos por preguntarnos qué importancia tienen los aspectos geográficos en la ocurrencia de desastres. Paralelamente, nos encontrábamos en un momento coyuntural porque tres años antes de la presentación de nuestra propuesta se habían producido las catastróficas inundaciones en la ciudad de Santa Fe. En ese entonces, una noticia publicada en el periódico local refería al traslado y posible relocalización de población asentada en la zona de bañados del río Salado hacia la zona de la Costa. También en simultáneo, un grupo de investigadores encabezados por la Dra. Claudia Natenzon estudiaba el caso santafesino como un paradigma de las responsabilidades políticas y sociales en la producción de los desastres. ${ }^{1}$

A partir de esa confluencia de sucesos decidimos profundizar en la temática y observamos que en la región, y particularmente en la zona costera, la problemática debía enfocarse, en principio, en el estudio de las dimensiones constitutivas del riesgo, esto es, en la identificación de las peligrosidades y la determinación de las condiciones de vulnerabilidad de la población. Al respecto, había numerosos estudios referidos al riesgo en comparación con aquellos vinculados a vulnerabilidad. Nos preguntamos entonces: ¿cómo estudiar la vulnerabilidad?

Emprendimos la búsqueda de un nexo con la comunidad, un actor que nos posibilitara encontrarnos y debatir estos temas "académicos" por fuera de la universidad. En esa etapa de discusión se trabajó de manera conjunta en la elaboración de una hoja de ruta con la organización CANOA. A partir de ello, se diseñó la propuesta.

De nuestra parte, el compromiso más importante era elaborar un diagnóstico de las condiciones del riesgo ambiental, con el convencimiento de que para poder estar más "preparados" ante la emergencia, como puede ser una inundación, la tarea está en desentramar cuáles son las condiciones de vida o estructurales de los grupos sociales afectados. Y profundizar los estudios de dichas condiciones es centrarse en el estudio de la vulnerabilidad social, porque se considera que una de las claves para comprender cómo se construyen los desastres es el estudio de los procesos sociales y, en particular, en cómo el modo de producción capitalista incide en la producción desigual de desastres en la sociedad. Las diferencias sociales -fundamentalmente las económicas- nos sitúan/exponen de un modo distinto y por tanto los desastres dejan de ser naturales para ser un constructo social.

Este cambio en la distinción implica además una modificación en las perspectivas de intervención frente a los desastres, puesto que se requiere pensar y actuar con estrategias preventivas que no solo estén sujetas a aspectos técnicos considerados necesarios pero

1) Ver la opinión de Natenzón (2003) en el diario Clarín: https://www.clarin.com/opinion/inundacion-causas-politicas-naturales_0_r1sIDKWIOtg.html. Para profundizar sobre la vasta producción que el equipo del Programa Interdisciplinarios de Recursos Naturales y Ambientes (PIRNA) del Instituto de Geografía Romualdo Ardisone (Facultad de Filosofía y Letras. Universidad de Buenos Aires) ha desarrollado, consultar: http://geografia.institutos.filo.uba.ar/pirna 
no suficientes, como, por ejemplo, el sistema de monitoreo de un río sino a la mejora de las condiciones sociales de la población expuesta.

La producción y reproducción de vulnerabilidad definen y determinan la magnitud de los efectos ante la presencia de una peligrosidad natural, por ello son las principales responsables de los procesos de desastre (García Acosta, 2005). Dialécticamente, sociedades con diferente grado de vulnerabilidad producen distintos espacios de riesgo: la situación de vulnerabilidad refuerza las condiciones de producción del riesgo (Cabré, Castro, Levrino, Schmidt y Visintini, 2021).

\section{¿Por qué estudiar la Costa santafesina?}

En primer lugar, la elección obedeció a que parte de los integrantes del equipo conocían las localidades. La falta de estudios sobre esta zona y la necesidad de "no olvidar" que la Costa era el área más afectada por las inundaciones fueron preocupaciones iniciales que movilizaron nuestro trabajo. En el análisis partimos del supuesto de que en el territorio convivían nuevos y viejos pobladores. Los pobladores más antiguos tenían conocimientos respecto de dinámicas del río y las inundaciones, lo que les permitía vincularse de una forma particular con el territorio. Por su parte, los nuevos residentes (que suponíamos iban in crescendo) no tenían dichos saberes. En tal sentido, dar cuenta de esto era una preocupación nuestra tanto en términos empíricos como metodológicos. Considerábamos necesario no caer en una "amnesia ambiental" (Davis, 1999), aspecto que advertimos por la ausencia de crecidas extraordinarias y por la presencia de la defensa contra inundaciones, que brindaba una sensación de seguridad a la población y sus actividades.

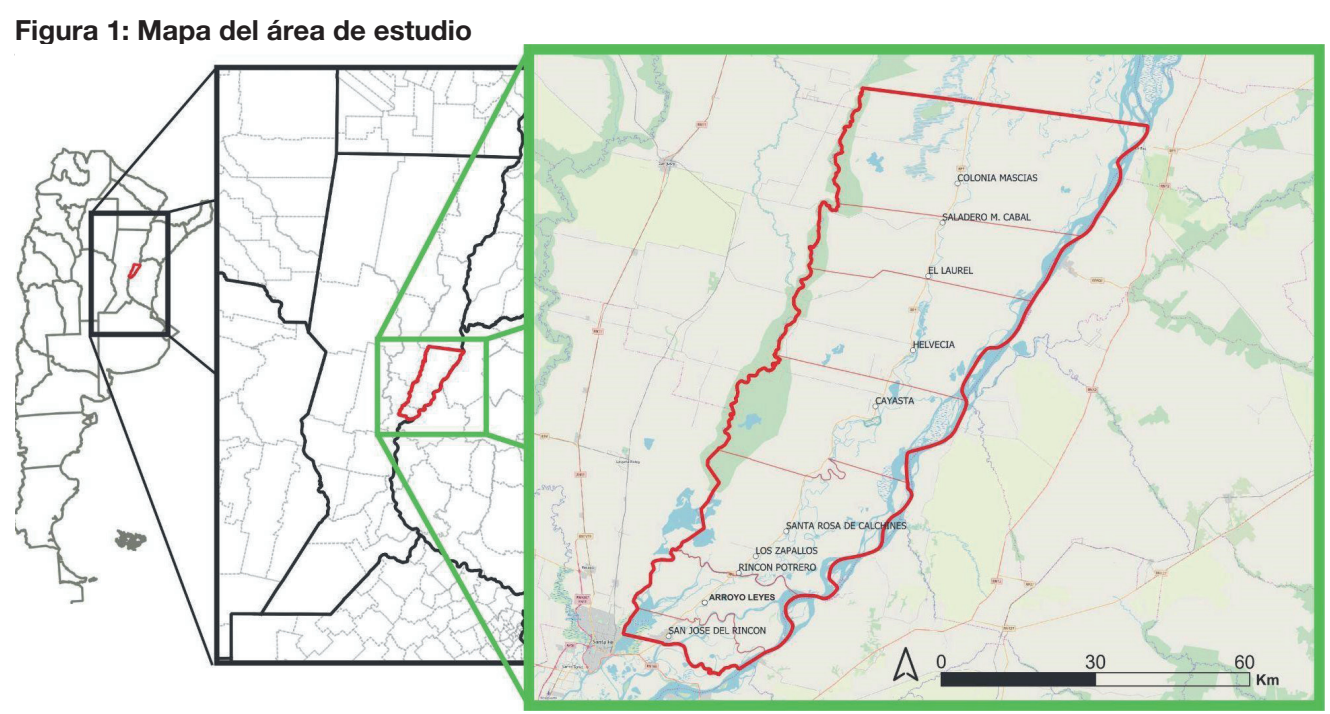

Fuente: elaboración propia.

A partir de ello comenzamos un trabajo de reconstrucción de las características del área para conocer las condiciones de vida de la población. Según datos de los Censos Naciona- 
les de Población, Hogares y Vivienda (CNPHyV), pudimos observar que, para el año 2001, los niveles del indicador Necesidades Básicas Insatisfechas (NBI) eran elevados: alrededor del $29 \%$ de la población del área, con valores distritales que oscilaban entre el $21 \%$ (en Rincón) y más del $40 \%$ (en Colonia Mascías y Saladero Cabal). Esos valores se encontraban muy por encima del promedio provincial, que era del 14,8 \%. Sin embargo, cabe destacar que la tendencia a lo largo del período 1991-2001 era decreciente: todos los distritos habían registrado un descenso en su población con NBI, tanto en volúmenes absolutos como en valores relativos. Esa variación, a su vez, estaba en consonancia con los valores promedio provinciales y nacionales, tanto absolutos como relativos, que habían disminuido a lo largo de esos diez años.

En este contexto consideramos oportuna la posibilidad de realizar un PEC porque a través de él podíamos avanzar tanto en la vinculación entre comunidad y universidad como en el diálogo entre los diferentes tipos de saberes. A su vez, creíamos que esta propuesta por parte de la cátedra podía propiciar la participación de estudiantes en otras instancias de la vida universitaria.

Ahora bien, a la pregunta que nos hicimos (¿por qué estudiar la Costa santafesina?) anexamos otra: ¿por qué hacer extensión en los pueblos de la Costa? Como afirma Menendez (2011), toda práctica de extensión supone la intervención sobre el territorio y la sociedad para el abordaje de una problemática que pone de manifiesto la presencia o ausencia del Estado. Asimismo, el autor sostiene:

"las políticas de Extensión ubican a la Universidad en diálogo permanente con las organizaciones de la sociedad civil y el Estado, contribuyendo a partir del conocimiento y el pensamiento crítico, al estudio, diseño, formulación, monitoreo y evaluación de políticas públicas". (Menéndez, 2011, p. 42)

Como hemos mencionado previamente, los pueblos de la Costa como territorio de extensión presentaban algunas problemáticas que en principio eran conocidas por el grupo extensionista pero necesitaban ser profundizadas y analizadas desde un marco teórico de referencia. Para poder dar cuenta de las dinámicas particulares que configuraban el riesgo a inundaciones se utilizó el enfoque proveniente de la Geografía de los Riesgos. A partir de la selección de dicho marco teórico, desarrollamos diferentes instancias para conocer en profundidad las condiciones del área de estudio. En este proceso, fue central la integración de la extensión con la investigación para lograr un acercamiento a las particularidades territoriales y seleccionar un enfoque de abordaje adecuado.

A partir de la actividad de extensión, pensamos en la posibilidad de tejer alianzas con organizaciones de la sociedad civil e instituciones educativas de las localidades que abordamos (CANOA, escuelas de enseñanza media y técnica). Esto nos permitió dar voz a diferentes actores sociales interactuantes en el territorio, lo cual colaboró a un proceso de extensión abonado por la comunicación dialógica a través del trabajo desde el respeto al otro y la empatía.

\section{Instancia previa de investigación: ¿desde qué marco teórico estudiamos la Costa?}

En una primera exploración sobre la cuestión, realizada sobre la base de bibliografía y entrevistas a informantes clave, pudimos reconocer y analizar un conjunto de procesos que 
participaban en la configuración de las condiciones de amenaza y vulnerabilidad actuales en el área. Algunos resultados obtenidos fueron incluso expuestos en congresos y publicados como artículos científicos en revistas (Combín, Schmidt y Visintini, 2010; Schmidt et al., 2011).

En esta instancia se identificaron tres tipos principales de amenazas hidrometeorológicas en el área, en ocasiones convergentes:

- Las inundaciones por desbordes, asociados a los pulsos cíclicos de crecidas del río, fundamentalmente por incremento de los aportes en las nacientes. Al respecto, cabe señalar la tendencia al aumento de la frecuencia de picos de crecida, así como la importancia de esos picos en cuanto a magnitud.

- Las inundaciones por anegamiento, vinculadas a la presencia de lluvias extremas y/o frecuentes en el área y las dificultades de percolación y/o escurrimiento. En este sentido, cabe destacar la tendencia al aumento de los montos pluviométricos en los últimos 20 años.

- La erosión de márgenes del cauce en sectores puntuales (como sucede en la localidad de Cayastá, en relación con el río San Javier).

En función de los factores de amenaza, debemos señalar que el sector de mayor peligrosidad actual es el sur (distritos Rincón y Leyes), en tanto presenta riesgo de trasvase de aguas hacia el oeste a través del arroyo Leyes y del sistema Setúbal, así como desde el oeste por el sistema Setúbal. También presenta problemas de anegamiento en los sectores defendidos, que son además los terrenos que registran las cotas más bajas. En el resto del área la posibilidad de inundaciones se vincula al derrame de crecidas del río San Javier (cada vez más controlados, al menos en las zonas de bajos naturales) y a los desbordes del sistema de lagunas del paleocauce por el oeste.

\section{¿Cómo definimos la vulnerabilidad?}

Definida como la capacidad de la población de anticipar, sobrevivir, asistir y recuperarse del impacto de una amenaza, para su estudio optamos por una estrategia de operacionalización centrada en la identificación de sus componentes o dimensiones. Con el enfoque puesto en poder dar cuenta de los aspectos materiales de las condiciones de vida de la población, acudimos a la información estadístico-censal disponible, en particular, como ya mencionamos, la provista por los CNPHyV 1991, 2001 y 2010. Primero, y como aproximación a la caracterización de situaciones de pobreza, se utilizó el índice de NBI en tanto indicador que mide situaciones de carencia estructural a partir de la consideración de cinco necesidades básicas (hacinamiento, calidad de la vivienda, condiciones sanitarias, asistencia escolar y capacidad de subsistencia) y, posteriormente, seleccionamos una serie de indicadores censales que, entendemos, permiten captar privación o precariedad y que, por tanto, pueden aproximarnos al grado de vulnerabilidad (sociodemográfica) de la población.

En una segunda etapa de la investigación se buscó avanzar en el análisis de la configuración de las vulnerabilidades sociales a través del empleo de diversas fuentes y técnicas, tanto cuantitativas como cualitativas.

El objetivo fue rescatar la visión desde adentro de las comunidades afectadas, lo que Hewitt denomina visiones "desde abajo" (Hewitt, 1996), porque "si el riesgo y el daño reflejan principalmente las condiciones sociales, será entonces necesario entender cómo las cosas funcionan y cómo la gente actúa, o se le permite actuar, en su contexto societal" (Hewitt, 
1996, p. 32). Además, hay que tener en cuenta que generalmente las sociedades expuestas a desastres han desarrollado formas de defenderse o "estrategias de sobrevivencia" (Blaikie, 1996) que es necesario conocer porque pueden configurar una base más efectiva para prepararse ante futuras crisis.

Para lograr los objetivos planteados, la propuesta fue trabajar con métodos cualitativos, concretamente con los "testimonios orales". Al indagar sobre la interpretación y percepción del riesgo y la vulnerabilidad a través de fuentes orales, nos orientamos indefectiblemente hacia la cuestión de la memoria de los habitantes con relación al lugar en el que viven y a las inundaciones. En este caso lo que interesaba era la percepción e interpretación en cuanto a la realidad actual, pero ello también involucró una mirada histórica, una apelación a la memoria para la elaboración de una narración sobre el pasado sobre la cual se pudiera comparar y definir la situación actual. Al respecto se consideraron importantes los aportes del denominado "giro lingüístico" y, en especial, el desarrollo de enfoques biográficos dentro de las ciencias sociales.

En la instancia de investigación fue clave recuperar algunas reflexiones en torno a la relevancia que tiene el trabajo de campo en Geografía. Para la Geografía Humana, el trabajo de campo cobra importancia al ser una de las instancias que permiten aproximarse al objeto de estudio. ${ }^{2}$ En nuestra experiencia en el territorio se combinaron dos de las formas que distingue Zusman (2011): el compromiso social (más ligado a las tradiciones radical-marxista y críticas) y el trabajo etnográfico (más ligado a la antropología).

\section{Instancias de extensión universitaria: ¿cómo articulamos diferentes actividades de extensión en territorio?}

En el apartado previo detallamos el proceso de investigación que conforma la antesala de las actividades de extensión en territorio y dimos cuenta del marco teórico que guio nuestro trabajo. En el presente apartado nos disponemos a detallar cuáles fueron las experiencias desarrolladas como grupo de extensión. Como ya hemos comentado en la introducción, el siguiente esquema se compone de dos instancias de extensión distintas.

2) A lo largo de la historia epistemológica, hubo diferentes formas de realizar esos trabajos que se vincularon con distintos tipos de tradiciones geográficas. Livingstone, 1991 plantea que la Geografía ha significado distintas cosas para distintas personas en distintas épocas y de esta manera, estas diversas concepciones constituyen la tradición disciplinar (Zusman, 2011). 
Figura 2: Instancias de extensión en pueblos de la Costa

\begin{tabular}{|c|c|c|c|}
\hline Marco institucional & Período & Actividades & Resultados \\
\hline \multirow{7}{*}{$\begin{array}{l}\text { Proyecto de } \\
\text { Extensión de Cátedra } \\
\text { (Introducción a la } \\
\text { Geografía) }\end{array}$} & \multirow[t]{7}{*}{2006 a 2008} & \multicolumn{2}{|c|}{ 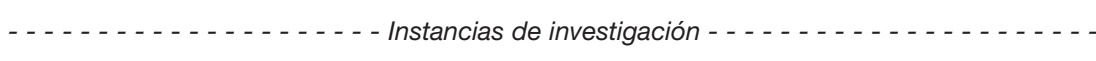 } \\
\hline & & $\begin{array}{l}\text { Estado de a cuestión: definición del marco } \\
\text { teórico, relevamiento de instituciones y } \\
\text { organizaciones de la sociedad civil vinculadas a } \\
\text { la temática en el área. }\end{array}$ & Informes, cuadros y mapas. \\
\hline & & \multicolumn{2}{|c|}{ - $\ldots$} \\
\hline & & $\begin{array}{l}\text { Reuniones con miembros de institución } \\
\text { interviniente (CANOA) }\end{array}$ & $\begin{array}{l}\text { Organización de taller en Santa Rosa } \\
\text { de Calchines con CANOA. }\end{array}$ \\
\hline & & $\begin{array}{l}\text { Imprementación del Taller sobre la percepción } \\
\text { de problemas ambientales del área con grupos } \\
\text { de población definidos por CANOA. }\end{array}$ & Análisis de los resultados del taller. \\
\hline & & $\begin{array}{l}\text { Participación del Ciclo de Talleres } \\
\text { "Vulnerabilidad Hídrica" en San José } \\
\text { del Rincón. }\end{array}$ & $\begin{array}{l}\text { Tejido de nuevos lazo, redes de trabajo } \\
\text { y colaboración. }\end{array}$ \\
\hline & & $\begin{array}{l}\text { Salida de campo: realización de entrevistas a } \\
\text { informantes clave, relevamiento en terreno y } \\
\text { ajuste de cartografía }\end{array}$ & $\begin{array}{l}\text { Segundo informe en base a resultados } \\
\text { de Taller y entrevistas. }\end{array}$ \\
\hline \multirow{7}{*}{$\begin{array}{l}\text { Programa de } \\
\text { Promoción de la } \\
\text { Universidad Argentina } \\
\text { "Un puente entre } \\
\text { la universidad y la } \\
\text { sociedad" }\end{array}$} & $\begin{array}{l}\text { Marzo a junio } \\
\text { de } 2009\end{array}$ & $\begin{array}{l}\text { Reuniones con directivos y docentes para } \\
\text { coordinar propuesta. } \\
\text { Instancias de taller: con alumnos y docentes } \\
\text { de la escuela. }\end{array}$ & $\begin{array}{l}\text { Mapas mentales del riesgo ambiental. } \\
\text { Informe y sistematización de talleres. }\end{array}$ \\
\hline & & - . . . . . . . . Discusión de resultados hacic & a el interior del equipo - . . . . . . . . . . . \\
\hline & Julio de 2009 & $\begin{array}{l}\text { Encuentro con alumnos y docentes } \\
\text { "La vuelta al aula". }\end{array}$ & Presentación y discusión de resultados. \\
\hline & & $\begin{array}{l}\text { Realización de entrevistas a familiares por parte } \\
\text { de alumnos de escuela media. } \\
\text { Realización de entrevistas a pobladores locales } \\
\text { por parte del equipo extensionista. }\end{array}$ & $\begin{array}{l}\text { Entrevistas a pobladores de San José } \\
\text { del Rincón y Santa Rosa de Calchines con } \\
\text { relación a su memoria de las inundaciones. }\end{array}$ \\
\hline & Enero de 2010 & $\begin{array}{l}\text { Finalización del proceso de relevamiento, } \\
\text { análisis y sistematización de datos, entrevistas } \\
\text { y material cartográfico }\end{array}$ & $\begin{array}{l}\text { Cuadernillo docente "El riesgo a } \\
\text { inundaciones en los Pueblos de la Costa } \\
\text { santafesina: una propuesta para el aula". }\end{array}$ \\
\hline & Año 2019-2020 & \multicolumn{2}{|c|}{$\begin{array}{l}\text { Actualización del trabajo: incorporación de miembros al equipo, construcción } \\
\text { de cartografía, trabajo de campo: relevamiento de cambios y continuidades. }\end{array}$} \\
\hline & Año 2021 & \multicolumn{2}{|c|}{$\begin{array}{l}\text { Publicación del libro: "Las condiciones de riesgo ambiental en los pueblos } \\
\text { de la Costa" (Ediciones UNL) }\end{array}$} \\
\hline
\end{tabular}

Fuente: elaboración propia.

\section{Las experiencias de extensión universitaria}

A continuación, expondremos los aspectos más importantes de los dos proyectos de extensión universitaria.

\section{PEC en el marco de la asignatura Introducción a la Geografía (2006-2008)}

La primera etapa se llevó a cabo a partir del PEC "Las condiciones de riesgo ambiental en los pueblos de la Costa". El mismo se propuso desde la cátedra de Introducción a la Geografía e involucró a las docentes a cargo y a un grupo de estudiantes que había cursado la asignatura recientemente, además de otros estudiantes avanzados de la carrera. 
En esta primera instancia, como se mencionó, la institución vinculante fue CANOA, una Asociación Civil y ONG de la ciudad de Santa Fe que venía trabajando desde hacía varios años en el territorio costero. Una de sus líneas de trabajo se vincula a la construcción del hábitat popular y desarrollo local a partir de la interacción de diferentes actores sociales y políticos. En ese sentido, nos pareció una articulación que podía enriquecer el desarrollo del proyecto.

De esta forma, comenzamos el diálogo y realizamos varios encuentros entre el equipo del proyecto y algunos de los integrantes de CANOA para socializar y discutir información relevante, aportes teóricos, y trazar un plan de trabajo. A estos encuentros se sumaron talleres que se desarrollaron en el territorio con el objetivo de intercambiar información y compartir nuestra propuesta con representantes de las instituciones locales $\mathrm{y}$, a la vez, identificar informantes clave, concertar entrevistas e indagar acerca de los roles de esas instituciones locales (gubernamentales y no gubernamentales), así como de actores individuales de la sociedad civil y de los organismos de gobierno en todos sus niveles.

El trabajo por parte del equipo del proyecto estuvo organizado en diferentes instancias: mantuvimos reuniones periódicas (cada 15 días) para socializar avances, elaborar la agenda de trabajo, plantear las problemáticas que iban surgiendo y pensar soluciones, discutir lecturas para seguir enriqueciendo y profundizando el marco teórico y organizar los encuentros con CANOA y los talleres en conjunto. En los intervalos entre talleres efectuamos tareas de relevamiento de información en organismos públicos, entrevistas, salidas de campo y reuniones reducidas que nos permitían avanzar en la investigación.

En términos de productos finales, se han elaborado un Informe Final sustantivo y dos Cartillas: una, con una síntesis de las problemáticas ambientales de las localidades del área, con cartografía propia, y otra con organigramas sobre la gestión del riesgo hídrico del área en sus diferentes escalas de competencia. Esto fue clave ya que la ausencia de información básica o la falta de sistematicidad y desactualización de aquella que estaba disponible fue uno de los problemas a resolver para la investigación y conocimiento del territorio. Debido a ello, gran parte de los esfuerzos se orientaron a relevar múltiples instituciones a través de entrevistas a funcionarios, visitas a dependencias de la gestión estatal, búsquedas en archivos hemerográficos y revisión bibliográfica, para luego organizar dicha información de acuerdo con los objetivos del trabajo.

\section{El Proyecto de Extensión de la Secretaría de Políticas Universitarias: un trabajo comparativo entre los distritos Rincón y Santa Rosa (Provincia de Santa Fe), para producir saberes}

En esta etapa, y por razones de operatividad, optamos por seleccionar algunos lugares en particular y desarrollar una estrategia (espacialmente) comparativa, lo que posibilitó indagar más abiertamente acerca de las diferencias geográficas en la construcción social del riesgo. Estos lugares son los distritos comunales San José de Rincón ${ }^{3}$ (en el departamento La Capi-

3) En el distrito comunal de San José de Rincón residían, al momento de realizar el trabajo, 8474 personas, y en el de Santa Rosa de Calchines, 5617 personas, según los datos del Censo Nacional de Población de 2001. La mayor parte de esa población habitaba en las localidades homónimas situadas en torno a la Ruta Provincial № 1, la zona más alta del albardón costero; el resto residía en localidades de menor tamaño y en unos pocos parajes rurales. Entre la población del área se evidenciaba una situación social bastante crítica: los valores de población con NBI estaban entre el 20 y el $30 \%$ del total de cada distrito, aunque existían zonas (y sectores sociales) en los que ese porcentaje ascendía a casi el $60 \%$. 
tal) y Santa Rosa de Calchines (en el departamento Garay). Esta elección se sustentó en los diferentes procesos de ocupación y aprovechamiento productivo del valle que registramos en el PEC. En San José del Rincón observamos una dinámica poblacional fuertemente vinculada a la ciudad de Santa $\mathrm{Fe}$, de cuya área metropolitana forma parte, y una organización productiva cada vez más centrada en los servicios (desde los turísticos hasta los gubernamentales). En Santa Rosa de Calchines, una historia demográfica y social asociada a grupos indígenas y colonos y un uso productivo cada vez más ligado a la producción agrícola intensiva. Ambos casos presentaban diferentes tipos de amenazas de inundaciones: por trasvasamiento de aguas desde el este y el oeste, en el primer caso, y solo por crecidas y desborde desde el este, principalmente, en el segundo caso.

Las preguntas que nos hacíamos entonces eran: ¿cómo perciben e interpretan el riesgo a inundaciones los pobladores del área? ¿Cuán vulnerables se sienten y por qué? ¿Qué representaciones/ significaciones tienen y han tenido los pobladores sobre las inundaciones y cómo viven con ellas? ¿Cómo las sufren, cómo las enfrentan? ¿Cómo las superan?

Para captar una mayor pluralidad de voces $y$, de esta manera, obtener diversas perspectivas, pensamos en un enfoque historicista y comprensivista para llevar adelante el trabajo. La Geografía ha evolucionado en forma contundente desde los años 70, en particular por la proliferación de nuevas voces que se reconocen en una Geografía que, en términos generales, podemos llamar "humanista". ${ }^{4}$ Así, elaboramos un esquema de entrevistas para interrogar a personas de ambas localidades que tuviesen diferentes edades, géneros, ocupaciones, tiempos de residencia en los lugares, entre otras variables. El vector que guio las

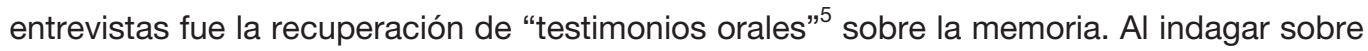
la interpretación y percepción del riesgo y la vulnerabilidad a través de fuentes orales, nos orientamos indefectiblemente hacia la cuestión de la memoria de los habitantes con relación al lugar en el que vivían y a las inundaciones.

Se entrevistó a población de la zona, a personas que vivían en las plantas urbanas y otras en la zona rural. Se entrevistó también a algunos representantes de la gestión estatal que tuvieron competencia en el área de interés. Toda esta tarea fue llevada adelante con el fin de producir y sistematizar información sobre peligrosidad natural, distribución y condiciones de vida de la población, actividades productivas y gestión territorial.

El análisis de estas entrevistas resultó un recurso muy valioso para enriquecer y dotar de otra "mirada" (como se refirió más arriba: una mirada "desde abajo") a la investigación realizada en la etapa anterior. Al mismo tiempo, algunos fragmentos de dichas entrevistas sirvieron como material para trabajar la problemática del riesgo con los estudiantes secundarios en los talleres llevados a cabo en las escuelas de ambas localidades.

En total se realizaron seis talleres: tres en la escuela de Educación Media Técnica № 658 María Sánchez de Thompson de San José del Rincón, y tres en la Escuela de Orientación Técnica Particular Incorporada $N^{\circ} 2020$ San Isidro Labrador. En estos encuentros se llevó

4) Hiernaux (2008) denomina a las "Geografías sensibles" a aquellas geografías que intentan incorporar al individuo, el rol de la persona como actor decisivo de su futuro, aun si su acción está enmarcada en una construcción social, lo que interesa es observar su papel en la producción social del espacio.

5) Es una metodología de tipo cualitativa que incorpora las fuentes orales y apela a la memoria individual, siempre entendida dentro de un marco social, porque interpreta que los mismos son portadores de la representación general de la sociedad, de sus necesidades y sus valores (Falchini, Hechim, 2003; Jelin, 2002; Catela, 2005; Pollak, 2006). 
adelante una propuesta didáctica en la que se trabajaron los conceptos centrales y aportes teóricos sobre la problemática del riesgo ambiental a partir del caso del Paraná Medio, la producción de mapas mentales acerca del riesgo en la escala local, las producciones de los estudiantes, sus percepciones y representaciones de y las de sus familias sobre esta problemática. También se hicieron "observaciones etnográficas" de cada uno de los tres talleres.

Tanto los talleres como la realización de las entrevistas por parte de los integrantes del equipo, fueron un insumo provechoso en función de los objetivos del proyecto. En primer lugar, porque ambas instancias implicaron un encuentro real con los pobladores $\mathrm{y}$, de este modo, el establecimiento de un vínculo. En segundo lugar, porque pudimos recabar qué conocimientos previos poseían sobre el tema y cómo los asociaban con su contexto inmediato y con el pasado. De este modo, se trató de lograr una reconstrucción histórica del riesgo pero poniendo énfasis en las condiciones actuales, es decir, en cuáles eran las percepciones que ellos tenían en el presente acerca de los riesgos naturales o tecnológicos en sus lugares, y cuáles las posibles amenazas latentes. También es importante destacar que, en particular y por la propia dinámica de taller, que permitió a los estudiantes dialogar libremente acerca de aquellas temáticas que se iban presentando en su transcurso, la propia discusión y conversación temática los motivaban para que profundizaran sus ideas en aquellos aspectos que considerábamos de mayor relevancia al objeto de investigación.

\section{Reflexiones finales}

Consideramos importante resaltar en el cierre de estas experiencias compartidas algunas cuestiones que tienen que ver con aspectos disciplinares y académicos. En principio, nos parece central remarcar que el trabajo realizado, como hemos evidenciado, se caracterizó por integrar instancias de investigación con instancias de extensión universitaria. En este sentido, tanto en momentos propios de la investigación como de la extensión, se produjo un intercambio de saberes que implicó constantemente poner en relación conocimientos académicos con conocimientos locales, de forma tal que no se establezcan relaciones de poder sino una integración horizontal de los mismos, contribuyendo así una producción colaborativa del conocimiento.

Respecto de la extensión, se torna relevante aquí recuperar algunas reflexiones sobre las etapas de extensión desarrolladas. Por un lado, el PEC se consolidó como una instancia que permitió formar estudiantes en la temática, donde los aspectos metodológicos y el trabajo de campo se afianzaron. A su vez, los cursantes de la asignatura se insertaron en la vida universitaria a partir de tareas de extensión.

Por otro lado, el Programa de Extensión Universitaria, como continuación del compromiso asumido por la cátedra, no solo representó una permanencia de nuestra presencia en territorio, sino la posibilidad de proseguir con la formación y participación del equipo de trabajo participante en la instancia previa. Al respecto, el trabajo sin interrupciones en territorio nos permitió conocer y seguir de cerca las dinámicas territoriales y tender nuevas redes de trabajo con otras instituciones (otros proyectos de investigación, escuelas medias de la zona, pobladores locales). En ese proceso fuimos capaces de reconocer el trabajo de extensión como productor de espacios e interrelaciones que se reflejaron en los lazos afianzados y las redes consolidadas, como también en la producción de documentos conjuntos como son 
cuadernillos docentes para trabajar la temática del riesgo en la escuela media o la reciente elaboración de un libro que tuvo como base los trabajos comenzados desde estas instancias de extensión, a las que se sumó una actualización de información.

\section{Referencias bibliográficas}

Blakie, P.; Cannon, T.; Davis, I. y Wisner, B. (1996). Vulnerabilidad: el entorno social, político y económico de los desastres. Tercer Mundo Editores.

Cabré, P; Castro, H; Levrino, J.P; Schmidt, C.G; Visintini, M.L (2021): Las condiciones del riesgo ambiental en los pueblos de la costa (sector sur). Colección Ciencia y Técnica. Editorial UNL. Santa Fe.

Castro, H.; Visintini, M.; Schmidt, C.; Combín, D.; Levrino, J. (2009). Aportes para una Geografía del riesgo ambiental. ¿Cómo captar las condiciones de vulnerabilidad social? XII Encuentro de Geógrafos de América Latina. Universidad de la República.

Combin, D.; Schmidt, C. y Visintini, M. (2010). La vulnerabilidad económico-productiva en el departamento Garay, Santa Fe: el caso de la ganadería de islas. Revista Universitaria de Geografía, 19, 19-47.

Davis, M. (1999). Ecology of Fear: Los Angeles and the Imagination of Disaster. Vintage Books.

Falchini, A.; Hechim, M. (2003). Contar la inundación. Ediciones UNL.

Hewitt, K. (1996). Daños ocultos y riesgos encubiertos: haciendo visible el espacio social de los desastres. En Mansilla, E. (Ed.). Desastres: modelo para armar. Colección de piezas de un rompecabezas social (pp. 11-29). La Red.

Hiernaux, N. (2008). "Geografía Objetiva" versus "Geografia Sensible": trayectorias divergentes de la Geografía Humana en el siglo XX. Revista da ANPEGE, 4(04), 27-38.

Jelin, E. (2002). Los trabajos de la memoria. Siglo XXI Editores.

Menéndez, G. (2011). Extensión y políticas públicas: la universidad en el centro del debate. $+E$ revista de Extensión Universitaria, 1(1). https://bibliotecavirtual.unl.edu.ar/publicaciones/index.php/Extension/article/ view/457

Natenzón, C. (2003, mayo 11). Una inundación tiene causas más políticas que naturales. Clarín. https://www. clarin.com/opinion/inundacion-causas-politicas-naturales_0_r1sIDKWIOtg.html

Pollack, M. (2006). Memoria, olvido, silencio. La producción social de identidades frente a situaciones límite. Ediciones Al Margen.

Visintini, M. L; Schmidt, C; Combín, D. (2009). La ganadería en tiempos de soja. Trayectorias productivas recientes en Garay (Pcia. de Santa Fe) y Las Heras (Pcia. de Buenos Aires). VI Jornadas interdisciplinarias de estudios agrarios y agroindustriales. Centro Interdisciplinario de Estudios Agrarios. Facultad de Ciencias Económicas. Universidad de Buenos Aires.

Visintini, M. L; Schmidt, C.; Levrino, J. (2010). Metodologías cualitativas y riesgo: los testimonios orales en el (re) conocimiento de la vulnerabilidad en los pueblos de la costa. IX Jornadas de Investigación en Geografía. FHUC-UNL.

Zusman, P. (2011). La tradición del trabajo de campo en Geografía. Geograficando, 7 (7), 15-32. 